\title{
Free $q$-Deformed Relativistic Wave Equations by Representation Theory
}

\author{
Christian Blohmann \\ Ludwig-Maximilians-Universität München, Sektion Physik \\ Lehrstuhl Prof. Wess, Theresienstr. 37, D-80333 München \\ Max-Planck-Institut für Physik, Föhringer Ring 6, D-80805 München
}

\begin{abstract}
In a representation theoretic approach a free $q$-relativistic wave equation must be such, that the space of solutions is an irreducible representation of the $q$-Poincare algebra. It is shown how this requirement uniquely determines the $q$-wave equations. As examples, the $q$-Dirac equation (including $q$-gamma matrices which satisfy a $q$-Clifford algebra), the $q$-Weyl equations, and the $q$-Maxwell equations are computed explicitly.
\end{abstract}

\section{Introduction}

Quantum field theories on noncommutative spaces have been receiving a tremendous amount of attention during the last few years (for reviews see [1, 2]) and, indeed, considerable progress was made. Noncommutative geometry has naturally appeared in a certain low energy limit of string theory 3] and gauge theories on general noncommutative geometries have found a solid, perturbative formulation [4,5] within the framework of deformation quantization. Expanding the product of noncommutative quantum fields perturbatively and relating the noncommutative gauge potentials and fields to their ordinary, commutative counterparts via the Seiberg-Witten map has put quantum theories on noncommutative spaces within the range of phenomenological considerations: A minimal noncommutative extension of the standard model was formulated [6], the effects of noncommutative geometry on magnetic and electric moments was studied [7,8], noncommutative neutrino-photon coupling with possible astrophysical implications was investigated [9, the OPAL collaboration has started looking for noncommutative signatures in electron positron pair annihilation [10], just to name some recent examples. For a review on the phenomenological implications of noncommutative geometry see [1].

Currently, most papers studied the particularly simple case where the commutator of the space-time observables $\left[X_{\mu}, X_{\nu}\right]=\theta_{\mu \nu}$ is a constant antisymmetric matrix. This kind of noncommutativity can be viewed as due to a constant 
background field. In string theory it can be attributed to a constant $B$-field on a D-brane. Clearly, a noncommutativity, which originates from a constant background field breaks Lorentz symmetry. It could be argued that if the noncommutativity parameters $\theta_{\mu \nu}$ are small, the violation of Lorentz symmetry is only small, too. However, on the level of regularization of loop diagrams, the noncommutativity leads to an interdependence of ultra-violet and infra-red cutoff scales [12,13]. Phenomenologically, this UV/IR mixing is problematic, as it seems to put even large scale Lorentz symmetry and weakened notions of locality of noncommutative quantum field theory into doubt [14]. But as yet, UV/IR mixing was investigated in detail only for the case of constant $\theta_{\mu \nu}$.

In a self-contained theory it would be reasonable to expect $\theta_{\mu \nu}$ to become a dynamical quantity itself, which transforms covariantly with respect to some (perhaps generalized) space-time symmetry. Since constant $\theta_{\mu \nu}$ does not allow for a perturbative deformation of Lorentz symmetry, one has to look for alternatives. In this context, it has been proposed repeatedly [4, 5] to investigate quantum spaces as standard examples for noncommutative geometries with generalized symmetries, quantum groups, which are controlled by the same parameters as the noncommutativity of the spaces. On quantum spaces, the changes induced by noncommutativity to physical concepts that are tied to space-time symmetry, such as energy-momentum conservation, Lorentz invariance, independence of in and out states etc. would be better integrated in the perturbative approach to noncommutative gauge theories. This would be an advantage for phenomenological considerations.

Another motivation to study noncommutative space-times with quantum group symmetries has emerged from the attempts to explain the observation [15] of cosmic rays of energy beyond the spectral cutoff (the Greisen-Zatsepin-Kuzmin limit) which is expected due to interaction with the cosmic microwave background. The often proposed explanation of such ultra high energy rays by vacuum dispersion relations, that is, the dependence of the speed of light on the wavelength, was shown by Amelino-Camelia to be reconcilable in principle with the observer independence of the laws of physics [16. This leads to a modification of special relativity by the assumption that there is not only an observer invariant velocity but also an observer invariant length, the Planck length. This proposition, now called doubly special relativity, has initiated a large number of active studies from both, the mathematical and the phenomenological viewpoint. (For an overview see [17.) Remarkably, many of the concrete realizations of doubly special relativity have led to quantum spaces with a quantum group Lorentz symmetry [18].

Mathematically, the construction of noncommutative gauge theories on quantum spaces is quite involved, since covariance with respect to the quantum symmetry must be preserved at every step of the construction. Previous work in dealing with $q$-deformations has studied realizations of quantum spaces by star products [19,20] and the calculation of the Seiberg-Witten map for Abelian gauge theory on the quantum plane in first order [21. Ultimately, we would like to es- 
tablish the Feynman rules for QED on quantum Minkowski space. Towards this goal, the free fermionic and bosonic propagators have to be determined. Propagators can be viewed as inverses or Green's functions of free wave equations. Hence, one of the first steps is to establish the free wave equations for fermions and gauge bosons. This is the purpose of the present paper.

Free elementary particles can be identified with irreducible representations of the Poincaré group [22] or, equivalently, the Poincaré algebra. The representations are realized by Wigner spinors, that is, on-shell wave functions with spin indices carrying representations of the little algebras. If we want to describe interactions where energy and momentum can be transfered from one particle onto another, we need to leave the mass shell. And we need a way to describe several particle types and their coupling in one common formalism.

This can be done by introducing Lorentz spinor wave functions. That is, tensor products of the algebra of functions on spacetime with a finite vector space containing the spin degrees of freedom, the whole space carrying a tensor representation of the Lorentz algebra. The additional mathematical structure we need in order to couple two wave functions is provided by the multiplication within the algebra of space functions. However, these Lorentz spinor representations are not irreducible. Therefore, only an irreducible subrepresentation can be the space of physical states. This subrepresentation is conveniently described as kernel of a linear operator $\mathbb{A}$, that is, we demand all physical states $\psi$ to satisfy the wave equation $\mathbb{A} \psi=0$.

This line of thought relies on the sole assumption that the Poincaré algebra describes the basic symmetry of spacetime. In this work we replace the Poincaré algebra by its $q$-deformation [23, which describes the basic symmetry of $q$-deformed spacetime. Then we construct $q$-wave equations proceeding in exactly the same way as in the undeformed case.

In 24] similar covariance arguments have been used to find wave equations for a certain deformation of the conformal symmetry algebra, which, however, does not contain the $q$-Poincaré algebra we consider here. In order to construct $q$ deformed relativistic wave equations various other methods have been proposed, based on $q$-Clifford algebras [25], $q$-deformed co-spinors [26], or differential calculi on quantum spaces [27, 28, 29], leading to mutually different results. While each approach may be justified in its own right, the situation as a whole is unsatisfactory since it should be possible to determine the wave equations uniquely as in the undeformed case [30] without needing any additional mathematical structure besides the $q$-Poincaré algebra and the basic apparatus of quantum mechanics.

Throughout this article, it is assumed that $q$ is a real number $q>1$. We will frequently use the abbreviations $\lambda=q-q^{-1}$ and [2] $=q+q^{-1}$. The lower case Greek letters $\mu, \nu, \sigma, \tau$ denote 4 -vector indices running through $\{0,-,+, 3\}$. Lower 4-indices are raised by $P^{\mu}:=\eta^{\mu \nu} P_{\nu}$ with the 4-metric $\eta^{\mu \nu}$ of Eq. (78) such that $S_{\mu} P^{\mu}$ is a scalar. The upper case Roman letters $A, B, C$ denote 3 -vector indices running through $\{-1,0,+1\}=\{-, 3,+\}$. A very short introduction 
to the $q$-Poincaré algebra is given in Appendix A Some more mathematical background information for this article has been compiled in [31].

\section{$2 \quad q$-Spinor Wave Functions}

\subsection{General $q$-Wave Equations}

We seek linear wave equations $\mathbb{A} \psi=0$, where $\mathbb{A}$ is a linear operator. For ker $\mathbb{A}$ to be a subrepresentation, the operator must satisfy

$$
\mathbb{A} \psi=0 \quad \Rightarrow \quad \mathbb{A} h \psi=0
$$

for all $q$-Poincaré transformations $h$. Depending on the particle type under consideration we might include charge and parity transformations. $\mathbb{A}$ is not unique since the wave equations for $\mathbb{A}$ and $\mathbb{A}^{\prime}$ must be considered equivalent as long as their solutions are the same, $\operatorname{ker}(\mathbb{A})=\operatorname{ker}\left(\mathbb{A}^{\prime}\right)$.

Ideally, $\mathbb{A}$ is a projection operator, $\mathbb{A}=\mathbb{P}$, with $\mathbb{P}^{2}=\mathbb{P}, \mathbb{P}^{*}=\mathbb{P}$. Condition (10) is then equivalent to

$$
[\mathbb{P}, h]=0
$$

for all $q$-Poincaré transformations $h$. Whether the wave equation is written with a projection is a matter of convenience. The Dirac equation is commonly written with such a projection which is determined uniquely (up to complement) by condition (2). For the Maxwell equations a projection can be found but yields a second order differential equation. For this reason, the Maxwell equations are

commonly described by a more general operator $\mathbb{A}$, which leads to a first order equation.

\section{$2.2 \quad q$-Lorentz Spinors}

We define a general, single particle $q$-Lorentz spinor wave function as element of the tensor product $\mathcal{S} \otimes \mathcal{X}$ of a finite vector space $\mathcal{S}=\mathbb{C}^{n}$ holding the spin degrees of freedom and the space of $q$-Minkowski space functions $\mathcal{X}=\mathbb{R}_{q}^{1,3}$ (App. $\mathrm{A}$ ).

Let $\left\{e_{i}\right\}$ be a basis of $\mathcal{S}$ transforming under a $q$-Lorentz transformation $h \in$ $\mathcal{H}=\mathcal{U}_{q}\left(\operatorname{sl}_{2}(\mathbb{C})\right)$ as $h \triangleright e_{j}=e_{i} \rho(h)^{i}{ }_{j}$, where $\rho: \mathcal{H} \rightarrow \operatorname{End}(\mathcal{S})$ is the representation map. Any spinor $\psi \in \mathcal{S} \otimes \mathcal{X}$ can be written as

$$
\psi=e_{j} \otimes \psi^{j}
$$

where $j$ is summed over and the $\psi^{j}$ are elements of $\mathcal{X}$. The action of $h \in \mathcal{H}$ on a spinor is the tensor action

$$
h \psi=\left(h_{(1)} \triangleright e_{j}\right) \otimes\left(h_{(2)} \triangleright \psi^{j}\right)=e_{i} \otimes \rho\left(h_{(1)}\right)^{i}{ }_{j}\left(h_{(2)} \triangleright \psi^{j}\right) .
$$


This tells us that the $\mathcal{X}$-valued components of $h \psi$ are given by

$$
(h \psi)^{i}=\rho\left(h_{(1)}\right)^{i}{ }_{j}\left(h_{(2)} \triangleright \psi^{j}\right) .
$$

The transformation of $\psi$ can easily be generalized to the case where $\mathcal{S}$ carries a tensor representation of two finite representations. For the components of spinors with two indices we would get

$$
(h \psi)^{i j}=\rho\left(h_{(1)}\right)^{i}{ }_{i^{\prime}} \rho^{\prime}\left(h_{(2)}\right)^{j}{ }_{j^{\prime}}\left(h_{(3)} \triangleright \psi^{i^{\prime} j^{\prime}}\right),
$$

where $\rho$ and $\rho^{\prime}$ are the representation maps of the first and second index, respectively.

Furthermore, we get spinors from the action of tensor operators. Let $T^{i}$ be a $\rho$-tensor operator with respect to the left Hopf adjoint action, $\operatorname{ad}_{\mathrm{L}} h \triangleright T^{i} \equiv$ $h_{(1)} T^{i} S\left(h_{(2)}\right)=\rho(S h)_{j}^{i} T^{j}$, and $\psi=e_{j} \otimes \psi^{j}$ a $\rho^{\prime}$-spinor. Let us define the components of a spinor $\phi^{i j}$ with two indices by

$$
\phi^{i j}:=\left(T^{i} \psi\right)^{j}
$$

How does this new array of wave functions $\phi^{i j}$ transform under $q$-Lorentz transformations? Letting $h$ act from the left, we find

$$
h \phi^{i j}=\rho\left(h_{(1)}\right)^{j}{ }_{j^{\prime}}\left(h_{(2)} \triangleright \phi^{i j^{\prime}}\right),
$$

that is, $h$ acts only on the index of the wave functions $\psi^{j}$. However, if we transform $\phi^{i j}$ by transforming $\psi$ inside Eq. (7),

$$
\begin{aligned}
\left(T^{i}(h \psi)\right)^{j} & =\left(\left(T^{i} h\right) \psi\right)^{j}=\left(h_{(2)}\left[\operatorname{ad}_{\mathrm{L}} S^{-1}\left(h_{(1)}\right) \triangleright T^{i}\right] \psi\right)^{j} \\
& =\left(\rho\left(h_{(1)}\right)_{i^{\prime}} h_{(2)} T^{i^{\prime}} \psi\right)^{j}=\rho\left(h_{(1)}\right)_{i^{\prime}} h_{(2)} \phi^{i^{\prime} j} \\
& =\rho\left(h_{(1)}\right)_{i^{\prime}} \rho^{\prime}\left(h_{(2)}\right)^{j}{ }_{j^{\prime}}\left(h_{(3)} \triangleright \phi^{i^{\prime} j^{\prime}}\right),
\end{aligned}
$$

we find that $\phi^{i j}$ transforms as a $\rho \otimes \rho^{\prime}$-spinor. Note, that for the last calculation the order in the tensor product $\mathcal{S} \otimes \mathcal{X}$ is essential. It would not have worked out as nicely if we had constructed the spinor space as $\mathcal{X} \otimes \mathcal{S}$. Chief examples of this construction would be the gauge term $P^{\mu} \phi$ of the vector potential $A^{\mu}$, or the derivatives of the vector potential $P^{\mu} A^{\nu}$ used to construct the electromagnetic field strength tensor $F^{\mu \nu}$.

\section{$2.3 \quad q$-Derivatives}

The $q$-Poincaré algebra is the Hopf semidirect product of the $q$-Lorentz algebra and the $q$-Minkowski algebra generated by the momentum 4-vector $P_{\mu}$ (Appendix). It becomes a Hopf algebra upon bosonisation [32. Within the braided 
tensor product, the coproduct of the momenta takes the natural form $\Delta\left(P^{\mu}\right)=$ $P^{\mu} \underline{\otimes} 1+1 \underline{\otimes} P^{\mu}$ or, explicitly,

$$
\begin{aligned}
\Delta\left(P^{\mu}\right) & :=P^{\mu} \otimes 1+\mathcal{R}^{-1}\left(\kappa \otimes P^{\mu}\right) \mathcal{R} \\
& =P^{\mu} \otimes 1+\kappa L_{+\nu}^{\mu} \otimes P^{\nu},
\end{aligned}
$$

where $\mathcal{R}=\mathcal{R}^{(1)} \otimes \mathcal{R}^{(2)}$ is the real universal $\mathcal{R}$-matrix of the $q$-Lorentz algebra, $\kappa$ is a group-like scaling operator, $P^{\mu} \kappa=q P^{\mu} \kappa$, which commutes with all Lorentz generators, and the $L$-matrix $L_{+\nu}^{\mu}:=\mathcal{R}^{(1)} \Lambda\left(\mathcal{R}^{(2)}\right)^{\mu}{ }_{\nu}$ is given by the 4 -vector representation of the second tensor factor of $\mathcal{R}$.

As in the undeformed case, we can assume that the spinorial degrees of freedom carry the trivial representation of the momentum algebra, that is, $P_{\mu} \triangleright e_{j}=$ $\varepsilon\left(P_{\mu}\right) e_{j}=0$ and $\kappa \triangleright e_{j}=\varepsilon(\kappa) e_{j}=e_{j}$. Hence, $P_{\mu}$ acts on a $\rho$-spinor as $P_{\mu} \psi=\rho\left(L_{+\nu}^{\mu}\right) e_{j} \otimes\left(P^{\nu} \triangleright \psi^{j}\right)$, that is,

$$
\left(P^{\mu} \psi\right)^{i}=\rho\left(L_{+\nu}^{\mu}\right)_{j}^{i}\left(P^{\nu} \triangleright \psi^{j}\right) .
$$

This equation yields a well defined action for both, the antireal and the real $\mathcal{R}$ matrix. For the following computations we will chose the antireal $\mathcal{R}$-matrix, for only then the action of the momenta is compatible with the $*$-structure. The $L$-matrix for this case has been calculated in [31. The results for the alternative choice of the real $\mathcal{R}$-matrix are given in the Appendix.

The action of $P^{\mu}$ on each component $\psi^{j} \in \mathcal{X}$ can be viewed as derivation within the algebra of $q$-Minkowski space functions $\mathcal{X}$,

$$
\partial^{\mu}:=1 \otimes \mathrm{i} P^{\mu}
$$

Now we can interpret an operator linear in the momenta as $q$-differential operator. If $C_{\mu}=C_{\mu} \otimes 1$ are operators that act on the spinor indices only,

$$
\text { i } C_{\mu} P^{\mu}=C_{\mu} \rho\left(L_{+\nu}^{\mu}\right) \partial^{\nu}=\tilde{C}_{\nu} \partial^{\nu}
$$

where

$$
\tilde{C}_{\nu}:=C_{\mu} \rho\left(L_{+\nu}^{\mu}\right)
$$

such that $\tilde{C}_{\nu}$ still acts on the spinor index only, while $\partial^{\nu}$ acts componentwise, so the two operators commute $\left[\tilde{C}_{\mu}, \partial^{\nu}\right]=0$. We will calculate the transformation $C_{\mu} \rightarrow \tilde{C}_{\mu}$ for particular representations below. Finally, we remark that for the mass Casimir we have within the spinor representation $P_{\mu} P^{\mu}=\mathcal{R}^{-1}(1 \otimes$ $\left.P_{\mu} P^{\mu}\right) \mathcal{R}=1 \otimes P_{\mu} P^{\mu}$, hence, $P_{\mu} P^{\mu}=-\partial_{\mu} \partial^{\mu}$. This means, that mass irreducibility for a spinor is the same as mass irreducibility for each component of the spinor. 


\subsection{Conjugate Spinors}

One of the effects of using Lorentz spinors is that the underlying representations can no longer be unitary, since there are no unitary finite representations of the non-compact Lorentz algebra - in the $q$-deformed as well as in undeformed case. However, we can introduce non-degenerate but indefinite bilinear forms playing the role of the scalar product. With respect to these pseudo scalar products the spinors carry $*$-representations, that is, the *-operation on the algebra side is the same as the pseudo adjoint on the operator side.

The problem of non-unitarity arises from the finiteness of the spin part $\mathcal{S}$ within the space of spinor wave functions $\mathcal{S} \otimes \mathcal{X}$, so we can assume that the wave function part $\mathcal{X}$ does carry a $*$-representation. It is then sufficient to redefine the scalar product on $\mathcal{S}$ only. Consider a $D^{(j, 0)}$ representation of $\mathcal{U}_{q}\left(\mathrm{sl}_{2}(\mathbb{C})\right)$ with orthonormal basis $\left\{e_{n}\right\}$. We want to define a pseudo scalar product by $\left(e_{m} \mid e_{n}\right):=A_{m n}$ such that

$$
\left(e_{m} \mid(g \otimes h) \triangleright e_{n}\right)=\left((g \otimes h)^{*} \triangleright e_{m} \mid e_{n}\right)
$$

for any $g \otimes h \in \mathcal{U}_{q}\left(\mathrm{sl}_{2}\right) \otimes \mathcal{U}_{q}\left(\mathrm{sl}_{2}\right) \cong \mathcal{U}_{q}\left(\mathrm{sl}_{2}(\mathbb{C})\right)$. For a pseudo scalar product we must suppose $A_{m n}$ to be a non-degenerate, hermitian, but not necessarily positive definite matrix. Inserting the definition of the pseudo scalar product, the pseudo-unitarity condition (15) reads

$$
\begin{aligned}
\left(e_{m} \mid(g \otimes h) \triangleright e_{n}\right) & =\left(e_{m} \mid e_{n^{\prime}} \rho^{j}(g)^{n^{\prime}}{ }_{n} \varepsilon(h)\right) \\
& =A_{m n^{\prime}} \rho^{j}(g)^{n^{\prime}}{ }_{n} \varepsilon(h) \\
& \stackrel{!}{=}\left((g \otimes h)^{*} \triangleright e_{m} \mid e_{n}\right) \\
& =\left(e_{m^{\prime}} \varepsilon\left(g^{*}\right) \rho^{j}\left(h^{*}\right)^{m^{\prime}}{ }_{m} \mid e_{n}\right) \\
& =A_{m^{\prime} n} \overline{\varepsilon\left(g^{*}\right) \rho^{j}\left(h^{*}\right)^{m^{\prime}}{ }_{m}} \\
& =A_{m^{\prime} n} \varepsilon(g) \rho^{j}(h)^{m}{ }_{m^{\prime}},
\end{aligned}
$$

where we have used that $(g \otimes h)^{*}=\mathcal{R}_{21}\left(h^{*} \otimes g^{*}\right) \mathcal{R}_{21}^{-1}\left[33\right.$ and $\varepsilon\left(\mathcal{R}_{[1]}\right) \mathcal{R}_{[2]}=1$. Traditionally, the pseudo scalar product is not described by a matrix $A_{m n}$ but by introducing a conjugate spinor basis $\left\{\bar{e}_{n}\right\}$ by

$$
\bar{e}_{m}:=e_{m^{\prime}} A_{m^{\prime} m} .
$$

Using (16) the conjugate basis turns out to transform as

$$
\begin{aligned}
(g \otimes h) \triangleright \bar{e}_{n} & =e_{m^{\prime}} \rho^{j}(g)^{m^{\prime}}{ }_{m} \varepsilon(h) A_{m n} \\
& =e_{m^{\prime}} A_{m^{\prime} n^{\prime}} \varepsilon(g) \rho^{j}(h)^{n^{\prime}}{ }_{n} \\
& =\bar{e}_{n^{\prime}} \varepsilon(g) \rho^{j}(h)^{n^{\prime}}{ }_{n},
\end{aligned}
$$

that is, $\bar{e}_{n}$ must transform according to a $D^{(0, j)}$ representation. $D^{(j, 0)}$ and $D^{(0, j)}$ being inequivalent representations, the conjugate basis $\bar{e}_{n}$ cannot be expressed as 
a linear combination of the original basis vectors $e_{n}$. In order to allow for a conjugate spinor basis we must consider a representation which contains both, $D^{(j, 0)}$ and $D^{(0, j)}$, and thus at least their direct sum $D^{(j, 0)} \oplus D^{(0, j)}$ as subrepresentation.

So far, it seems that everything is almost trivially analogous to the undeformed case. It is not. If we consider irreducible representations of mixed chirality, $D^{(i, j)}$, we find that the appearance of the $\mathcal{R}$-matrix in $(g \otimes h)^{*}$ makes it impossible to define conjugate spinors. It only works for $D^{(j, 0)}$, because $\rho^{0}=\varepsilon$ and $\varepsilon\left(\mathcal{R}_{[1]}\right) \mathcal{R}_{[2]}=$ 1. Fortunately, we do have conjugate spinors for the most interesting cases: Dirac spinors $\left(D^{\left(\frac{1}{2}, 0\right)} \oplus D^{\left(0, \frac{1}{2}\right)}\right)$ and the Maxwell tensor $\left(D^{(1,0)} \oplus D^{(0,1)}\right)$. For these cases everything is analogous to the undeformed case.

Let us consider a $D^{(j, 0)} \oplus D^{(0, j)}$ representation with basis $\left\{e_{n}^{\mathrm{L}}\right\}$ for the left chiral subrepresentation $D^{(j, 0)}$ and the basis $\left\{e_{n}^{\mathrm{R}}\right\}$ for $D^{(0, j)}$. We define the conjugate basis by $\overline{e_{n}^{\mathrm{L}}}:=e_{n}^{\mathrm{R}}$ and $\overline{e_{n}^{\mathrm{R}}}=e_{n}^{\mathrm{L}}$. Let us call $\mathcal{P}$ the parity operator that exchanges the left and right chiral part. Its matrix representation with respect to the basis $\left\{e_{n}^{\mathrm{L}}, e_{n}^{\mathrm{R}}\right\}$ is

$$
\mathcal{P}_{m n}=\left(\begin{array}{ll}
0 & 1 \\
1 & 0
\end{array}\right),
$$

where 1 is the $(2 j+1)$-dimensional unit matrix. This is the matrix that represents our new pseudo scalar product as a bilinear form. The pseudo Hermitian conjugate of some operator $A$ can now be written as

$$
j(A):=\mathcal{P} A^{\dagger} \mathcal{P},
$$

which is an involution because $\mathcal{P}^{\dagger}=\mathcal{P}^{-1}$ and an algebra anti-homomorphism because $\mathcal{P}=\mathcal{P}^{-1}$.

We apply this result to the whole space of spinor wave functions $\mathcal{S} \otimes \mathcal{X}$. Let us assume that the scalar product of two wave functions $f, g \in \mathcal{X}$ can be written (at least formally) as some sort of integral $\langle f \mid g\rangle=\int f^{*} g$. The pseudo scalar product of two $D^{(j, 0)} \oplus D^{(0, j)}$ spinors $\psi$, $\phi$ becomes

$$
\begin{aligned}
(\psi \mid \phi) & =\left(e_{m} \otimes \psi^{m} \mid e_{n} \otimes \phi^{n}\right)=\left(e_{m} \mid e_{n}\right)\left\langle\psi^{m} \mid \phi^{n}\right\rangle \\
& =\int\left(\psi^{m}\right)^{*} \mathcal{P}_{m n} \phi^{n}=\int \bar{\psi}^{n} \phi^{n},
\end{aligned}
$$

with the conjugate spinor wave function defined as

$$
\bar{\psi}^{n}:=\left(\psi^{m}\right)^{*} \mathcal{P}_{m n} .
$$

In summary, we have convinced ourselves that in the case of $D^{(j, 0)} \oplus D^{(0, j)}$ representations the conjugation of spinors, of spinor wave functions, and of operators works exactly as in the undeformed case. 


\section{The $q$-Dirac Equation}

\subsection{The $q$-Dirac Equation for the Rest States}

In this section we consider $q$-Dirac spinors $\psi=e_{i} \otimes \psi^{i}$ with the spin part transforming according to a $D^{\left(\frac{1}{2}, 0\right)} \oplus D^{\left(0, \frac{1}{2}\right)}$ representation [31. We want to write the $q$-Dirac equation as expression which involves momenta only to first order, corresponding to a first order differential equation

$$
\mathbb{P} \psi:=\frac{1}{2 m}\left(m+\gamma_{\mu} P^{\mu}\right) \psi=0,
$$

with the $\gamma_{\mu}$ being some operators acting on $\psi^{i}$. We can already say that $\gamma_{\mu}$ must be a left 4 -vector operator. If it were not, $\gamma_{\mu} P^{\mu}$ would not be scalar and, hence, would not commute with the $q$-Lorentz transformations as required in Eq. (21).

We consider here a massive $q$-Dirac spinor representation, so there is a set of rest states $\psi$ which the momenta act upon as $P^{0} \psi=m \psi, P^{A} \psi=0$ 34. We start the search for a projection $\mathbb{P}$ that reduces the $q$-Dirac representation by computing how it acts on these rest states, where we have

$$
\mathbb{P}_{0}=\frac{1}{2}\left(1+\gamma_{0}\right),
$$

the zero indicating that $\mathbb{P}_{0}$ acts on the rest states only. We assume that we can realize the operator $\gamma_{0}$ as $4 \times 4$-matrix that acts on the spin degrees of freedom only. This is not unreasonable, for if $\gamma_{\mu}$ is a set of matrices that form a 4 -vector operator in the $D^{\left(\frac{1}{2}, 0\right)} \oplus D^{\left(0, \frac{1}{2}\right)}$ representation then $\gamma_{\mu} \otimes 1$ will also be a 4 -vector operator in the representation of spinor wave functions. So let us assume we can write $\mathbb{P}_{0}=\mathbb{P}_{0} \otimes 1$ in block form as $\mathbb{P}_{0}=\left(\begin{array}{c}A \\ C\end{array}\right)$, where $A, B, C, D$ are $2 \times 2$-matrices.

The restriction of condition (2) to the rest states means that $\mathbb{P}_{0}$ must commute with the little algebra. The little algebra for the massive case is the $\mathcal{U}_{q}\left(\mathrm{su}_{2}\right)$ subalgebra of rotations [34]. A rotation $l \in \mathcal{U}_{q}\left(\mathrm{su}_{2}\right)$ is represented by

$$
\rho(l)=\left(\begin{array}{cc}
\rho^{\frac{1}{2}}(l) & 0 \\
0 & \rho^{\frac{1}{2}}(l)
\end{array}\right) .
$$

Since the $\rho^{\frac{1}{2}}$ representations of the rotations generate all $2 \times 2$-matrices (the $q$ Pauli matrices are a basis), $\mathbb{P}_{0}$ will only commute with all rotations if $A, B, C$, $D$ are numbers, that is, complex multiples of the unit matrix.

Furthermore, $\mathbb{P}_{0}$ has to be a projection operator, $\mathbb{P}_{0}^{2}=\mathbb{P}_{0}, \mathbb{P}_{0}^{\dagger}=\mathbb{P}_{0}$, and, as in the undeformed case, we require it to commute with the parity operator, $\left[\mathbb{P}_{0}, \mathcal{P}\right]=0$. Together these conditions fix $\mathbb{P}_{0}$ and hence $\gamma_{0}$ uniquely to

$$
\gamma_{0}=\left(\begin{array}{ll}
0 & 1 \\
1 & 0
\end{array}\right)
$$

the same as in the undeformed case. 


\subsection{The $q$-Gamma Matrices and the $q$-Clifford Algebra}

If $\gamma_{0}$ is to be a 4 -vector operator, we have to define the other gamma matrices as in Eq. (81) by

$$
\begin{aligned}
& \gamma_{-}=\operatorname{ad}_{\mathrm{L}}\left(-q^{-\frac{1}{2}} \lambda^{-1}[2]^{\frac{1}{2}} c\right) \triangleright \gamma_{0} \\
& \gamma_{+}=\operatorname{ad}_{\mathrm{L}}\left(q^{\frac{1}{2}} \lambda^{-1}[2]^{\frac{1}{2}} b\right) \triangleright \gamma_{0} \\
& \gamma_{3}=\operatorname{ad}_{\mathrm{L}}\left(\lambda^{-1}(d-a)\right) \triangleright \gamma_{0},
\end{aligned}
$$

where the adjoint action is understood with respect to the $q$-Dirac representation. In order to compute this explicitly, we have to calculate the representations of the boosts (75) first.

$$
\begin{aligned}
\rho(a) & =\left(\begin{array}{cc}
\rho^{\frac{1}{2}}\left(K^{\frac{1}{2}}\right) & 0 \\
0 & \rho^{\frac{1}{2}}\left(K^{-\frac{1}{2}}\right)
\end{array}\right) \\
\rho(b) & =\left(\begin{array}{cc}
0 & 0 \\
0 & q^{-\frac{1}{2}} \lambda \rho^{\frac{1}{2}}\left(K^{-\frac{1}{2}} E\right)
\end{array}\right) \\
\rho(c) & =\left(\begin{array}{cc}
-q^{\frac{1}{2}} \lambda \rho^{\frac{1}{2}}\left(F K^{\frac{1}{2}}\right) & 0 \\
0 & 0
\end{array}\right) \\
\rho(d) & =\left(\begin{array}{cc}
\rho^{\frac{1}{2}}\left(K^{-\frac{1}{2}}\right) & 0 \\
0 & \rho^{\frac{1}{2}}\left(K^{\frac{1}{2}}\right)
\end{array}\right)
\end{aligned}
$$

This gives us for example

$$
\begin{aligned}
\gamma_{+} & =\operatorname{ad}_{\mathrm{L}}\left(q^{\frac{1}{2}} \lambda^{-1}[2]^{\frac{1}{2}} b\right) \triangleright \gamma_{0} \\
& =q^{\frac{1}{2}} \lambda^{-1}[2]^{\frac{1}{2}}\left[\rho(b) \gamma_{0} \rho(a)-q \rho(a) \gamma_{0} \rho(b)\right] \\
& =[2]^{\frac{1}{2}}\left[\left(\begin{array}{cc}
0 & 0 \\
\rho^{\frac{1}{2}}\left(K^{-\frac{1}{2}} E K^{\frac{1}{2}}\right) & 0
\end{array}\right)-q\left(\begin{array}{cc}
0 & \rho^{\frac{1}{2}}(E) \\
0 & 0
\end{array}\right)\right] \\
& =\left(\begin{array}{cc}
0 & q \sigma_{+} \\
-q^{-1} \sigma_{+} & 0
\end{array}\right),
\end{aligned}
$$

where $\sigma_{+}$is one of the $q$-Pauli matrices defined as $q$-Clebsch-Gordan coefficients or, equivalently, as spin- $\frac{1}{2}$ representation of the angular momentum generators, $\sigma_{A}=[2] \rho^{\frac{1}{2}}\left(J_{A}\right)$ 31]. The analogous calculations for $\gamma_{-}$and $\gamma_{+}$yield

$$
\gamma_{0}=\left(\begin{array}{ll}
0 & 1 \\
1 & 0
\end{array}\right), \quad \gamma_{A}=\left(\begin{array}{cc}
0 & q \sigma_{A} \\
-q^{-1} \sigma_{A} & 0
\end{array}\right)
$$

where $A$ runs as usual through $\{-,+, 3\}$.

This result can be easily generalized to higher spin. All we have to do for a massive $D^{(j, 0)} \oplus D^{(0, j)}$-spinor is to replace $\rho^{\frac{1}{2}}$ with $\rho^{j}$. The result is higher dimensional $\gamma$-matrices

$$
\gamma_{0}^{(j)}=\left(\begin{array}{ll}
0 & 1 \\
1 & 0
\end{array}\right), \gamma_{A}^{(j)}=[2]\left(\begin{array}{cc}
0 & q \rho^{j}\left(J_{A}\right) \\
-q^{-1} \rho^{j}\left(J_{A}\right) & 0
\end{array}\right) .
$$


If we want to write the $q$-Dirac equation as $q$-differential equation, we need to calculate $\tilde{\gamma}_{\mu}$ by formula (14). For the $q$-Pauli matrices we get

$$
\begin{aligned}
& \sigma_{A} \rho^{\left(\frac{1}{2}, 0\right)}\left(\left(L_{\mathrm{I}+}^{\Lambda}\right)^{A}{ }_{B}\right)=q^{2} \tilde{\sigma}_{B} \\
& \sigma_{A} \rho^{\left(0, \frac{1}{2}\right)}\left(\left(L_{\mathrm{I}+}^{\Lambda}\right)^{A}{ }_{B}\right)=q^{-2} \tilde{\sigma}_{B},
\end{aligned}
$$

where we have used a variant of the $q$-Pauli matrices, defined as $\tilde{\sigma}_{A}:=-[2] \rho^{\frac{1}{2}}\left(S J_{A}\right)$. Explicitly, these are

$$
\tilde{\sigma}_{-}=[2]^{\frac{1}{2}}\left(\begin{array}{cc}
0 & q^{\frac{1}{2}} \\
0 & 0
\end{array}\right), \tilde{\sigma}_{+}=[2]^{\frac{1}{2}}\left(\begin{array}{cc}
0 & 0 \\
-q^{-\frac{1}{2}} & 0
\end{array}\right), \tilde{\sigma}_{3}=\left(\begin{array}{cc}
-q^{-1} & 0 \\
0 & q
\end{array}\right)
$$

with respect to the $\{-,+\}$ basis. For the transformed $q$-gamma matrices we thus obtain

$$
\tilde{\gamma}_{0}=\left(\begin{array}{ll}
0 & 1 \\
1 & 0
\end{array}\right), \quad \tilde{\gamma}_{A}=\left(\begin{array}{cc}
0 & q^{-1} \tilde{\sigma}_{A} \\
-q \tilde{\sigma}_{A} & 0
\end{array}\right),
$$

and the $q$-Dirac equation written as $q$-differential equation becomes

$$
\left(m-\mathrm{i} \tilde{\gamma}_{\mu} \partial^{\mu}\right) \psi=0 .
$$

After lengthy calculations we find that the gamma matrices satisfy the relations

$$
\tilde{\gamma}_{\sigma} \tilde{\gamma}_{\tau}=\eta_{\tau \sigma}+\tilde{\gamma}_{\mu} \tilde{\gamma}_{\nu} \mathbb{P}_{\mathrm{A} \tau \sigma}^{\nu \mu} \quad \Leftrightarrow \quad \tilde{\gamma}_{\mu} \tilde{\gamma}_{\nu} \mathbb{P}_{\mathrm{S} \sigma \tau}^{\nu \mu}=\eta_{\sigma \tau},
$$

where $\mathbb{P}_{\mathrm{A}}$ is the $q$-antisymmetrizer and $\mathbb{P}_{\mathrm{S}}=1-\mathbb{P}_{\mathrm{A}}$ is the $q$-symmetrizer of the Clebsch-Gordan series (76). This is the $q$-deformation of the Clifford algebra relations, from which now follows that the square of $q$-Dirac operator is indeed the mass Casimir,

$$
\left(\tilde{\gamma}_{\mu} \partial^{\mu}\right)^{2}=\partial_{\mu} \partial^{\mu}=-P_{\mu} P^{\mu} .
$$

As in the undeformed case we conclude that a solution $\psi$ to the $q$-Dirac equation satisfies automatically the mass shell condition $P_{\mu} P^{\mu} \psi=m^{2} \psi$, and that $\mathbb{P}=$ $\frac{1}{2 m}\left(m+\gamma_{\mu} P^{\mu}\right)$ really is a projection operator.

One could have started directly from relations (35) trying to find matrices that satisfy them [25]. This approach has a number of disadvantages: a) It is computationally much more cumbersome than boosting $\gamma_{0}$. b) The result is not unique, that is, we would get many solutions to the $q$-Clifford algebra not knowing which representations they belong to. c) Having determined a solution $\tilde{\gamma}_{\mu}$, the covariance of the $q$-Dirac equation remains unclear as $\tilde{\gamma}_{\mu}$ cannot be a 4 -vector operator.

\subsection{The Zero Mass Limit and the $q$-Weyl Equations}

The zero mass limit of the $q$-Dirac equation is formally

$$
\mathbb{A} \psi:=\gamma_{\mu} P^{\mu} \psi=0,
$$


where $\mathbb{A}$ is no longer a projection operator. The wave equation is now decoupled into two independent equations for a left handed $D^{\left(\frac{1}{2}, 0\right)}$-spinor $\psi_{\mathrm{L}}$ and a right handed $D^{\left(0, \frac{1}{2}\right)}$-spinor $\psi_{\mathrm{R}}$,

$$
\sigma_{A} P^{A} \psi_{\mathrm{L}}=q^{-1} P^{0} \psi_{\mathrm{L}}, \quad \sigma_{A} P^{A} \psi_{\mathrm{R}}=-q P^{0} \psi_{\mathrm{R}},
$$

the $q$-Weyl equations for massless left and right handed spin- $\frac{1}{2}$ particles. Written as $q$-differential equation they become

$$
\tilde{\sigma}_{A} \partial^{A} \psi_{\mathrm{L}}=-q \partial^{0} \psi_{\mathrm{L}}, \quad \quad \tilde{\sigma}_{A} \partial^{A} \psi_{\mathrm{R}}=q^{-1} \partial^{0} \psi_{\mathrm{R}}
$$

The operator $\mathbb{A}$ inherits property (11) from $\mathbb{P}$, so $\mathbb{A} \psi=0$ is a viable wave equation. On the massless momentum eigenspace [34] where $\left(P_{0}, P_{-}, P_{+}, P_{3}\right)=(k, 0,0, k)$, $\mathbb{A}$ acts as $\mathbb{A}_{0}=k\left(\begin{array}{cc}0 & 1-q \sigma_{3} \\ 1+q^{-1} \sigma_{3} & 0\end{array}\right)$. The kernel of this operator is 2-dimensional, the solution states corresponding to helicity $\pm \frac{1}{2}$.

If we generalize these considerations to higher spin $D^{(j, 0)} \otimes D^{(0, j)}$ Dirac type spinors, we find that the corresponding operator $\mathbb{A}$ has zero kernel, so the space of solutions is trivial. This applies in particular to $q$-Maxwell spinors. Therefore, we need a different approach to find the $q$-Maxwell equations - in complete analogy to the undeformed case [30].

\section{The $q$-Maxwell Equations}

\subsection{The $q$-Maxwell Equations in the Momentum Eigen- spaces}

In this section we consider massless $D^{(1,0)} \oplus D^{(0,1)}$ spinors. According to the Clebsch-Gordan series (76) this type of spinor is equivalent to a $q$-antisymmetric tensor $F^{\mu \nu}$ with two 4 -vector indices. These are the types of spinors commonly used to describe the electromagnetic field.

We start our calculations in the massless momentum eigenspace with momentum eigenvalues $\left(P_{0}, P_{-}, P_{+}, P_{3}\right)=(k, 0,0, k)$ for some real parameter $k$. It has been shown in 34 that this eigenspace is invariant under the little algebra generated by the group-like generator of $q$-rotations around the $z$-axis $K$ and

$$
N_{-}:=q^{\frac{1}{2}}[2]^{\frac{1}{2}} a c, N_{+}:=q^{\frac{1}{2}}[2]^{\frac{1}{2}} b d, N_{3}:=1+[2] b c .
$$

The irreducible $*$-representations of this algebra are one-dimensional, given by $K=\kappa=N_{3}$ and $N_{ \pm}=0$ for real $\kappa$. Within the momentum eigenspace the little algebra acts only on the spinor index, here, by the $D^{(1,0)} \oplus D^{(0,1)}$ matrix representation

$$
\begin{gathered}
K=\left(\begin{array}{cc}
\rho^{1}(K) & 0 \\
0 & \rho^{1}(K)
\end{array}\right), \quad N_{-}=-q[2]\left(\begin{array}{cc}
\rho^{1}\left(J_{-}\right) & 0 \\
0 & 0
\end{array}\right) \\
N_{+}=-q^{-1}[2]\left(\begin{array}{cc}
0 & 0 \\
0 & \rho^{1}\left(J_{+}\right)
\end{array}\right), \quad N_{3}=\left(\begin{array}{ll}
1 & 0 \\
0 & 1
\end{array}\right)
\end{gathered}
$$


where $\rho^{1}$ is the vector representation of $\mathcal{U}_{q}\left(\mathrm{su}_{2}\right)$. We seek an operator $\mathbb{P}_{0}=\mathbb{P}_{0} \otimes 1$ that projects onto an irreducible subrepresentation of the momentum eigenspace. As before, we write it in block form as $\mathbb{P}_{0}=\left(\begin{array}{c}A \\ C\end{array}\right)$ $3 \times 3$-matrices. We must have $\mathbb{P}_{0}^{\dagger}=\mathbb{P}_{0}$, so $A$ and $D$ must be Hermitian matrices and $C=B^{\dagger}$. Within an irreducible representation of the little algebra we have $N_{ \pm}=0$, so we must demand $N_{ \pm} \mathbb{P}_{0}=0$. This leads to the conditions

$$
\begin{aligned}
\rho^{1}\left(J_{-}\right) A=0, & \rho^{1}\left(J_{+}\right) D=0 \\
\rho^{1}\left(J_{-}\right) B=0, & \rho^{1}\left(J_{+}\right) B^{\dagger}=0 .
\end{aligned}
$$

To satisfy these conditions $A, B$, and $D$ must be of the form

$$
A=\left(\begin{array}{ccc}
\alpha & 0 & 0 \\
0 & 0 & 0 \\
0 & 0 & 0
\end{array}\right), \quad B=\left(\begin{array}{ccc}
0 & 0 & \beta \\
0 & 0 & 0 \\
0 & 0 & 0
\end{array}\right), \quad D=\left(\begin{array}{ccc}
0 & 0 & 0 \\
0 & 0 & 0 \\
0 & 0 & \delta
\end{array}\right),
$$

for $\alpha, \delta$ real and $\beta$ complex. Furthermore, $\mathbb{P}_{0}$ must project onto an eigenvector of $K$. From this it follows that $\beta=0$ and either $\alpha=1, \delta=0$ or $\alpha=0, \delta=1$. To summarize, there are two possible projections

$$
\mathbb{P}_{\mathrm{L}}=\left(\begin{array}{cccc}
1 & & & \\
& 0 & & \\
& & \backslash & \\
& & & 0
\end{array}\right), \quad \quad \mathbb{P}_{\mathrm{R}}=\left(\begin{array}{llll}
0 & & & \\
& \backslash & & \\
& & 0 & \\
& & & 1
\end{array}\right)
$$

projecting each on a irreducible one-dimensional representation of the little algebra. The image of $\mathbb{P}_{\mathrm{L}}$ is part of the left handed $D^{(1,0)}$ component while $\mathbb{P}_{\mathrm{R}}$ projects onto the right handed $D^{(0,1)}$ component of the spinor. Physically, this corresponds to left and right handed circular waves. We want to allow for parity transformations exchanging the left and right handed parts, so we need both parts $\mathbb{P}_{0}=\mathbb{P}_{\mathrm{L}}+\mathbb{P}_{\mathrm{R}}$. With the parity transformation included, the two dimensional space which $\mathbb{P}_{0}$ projects onto is irreducible.

\subsection{Computing the $q$-Maxwell Equations}

We want to write the $q$-Maxwell equations in the form of a first order differential equation

$$
\mathbb{A} \psi:=C_{\mu} P^{\mu} \psi=0,
$$

hoping that again the operators $C_{\mu}$ can be chosen to act on the spinor index only, $C_{\mu}=C_{\mu} \otimes 1$. Recall from the last section, that as long as we do not include parity transformations, we must have two independent equations for the right and the left handed part of the spinor, $\psi_{\mathrm{L}}$ carrying a $D^{(1,0)}$ representation and $\psi_{\mathrm{R}}$ carrying a $D^{(0,1)}$ representation

$$
\mathbb{A}_{\mathrm{L}} \psi_{\mathrm{L}}=0, \quad \mathbb{A}_{\mathrm{R}} \psi_{\mathrm{R}}=0 .
$$


For condition (1I) it would be sufficient (but not necessary) if $\mathbb{A}_{L}, \mathbb{A}_{R}$ were scalar operators. Let us try to choose $\mathbb{A}_{\mathrm{L}}=C_{\mu}^{\mathrm{L}} P^{\mu}$ and $\mathbb{A}_{\mathrm{R}}=C_{\mu}^{\mathrm{R}} P^{\mu}$ to be scalars with respect to rotations. For this to be possible $C_{0}^{\mathrm{L}}, C_{0}^{\mathrm{R}}$ must be scalars with respect to rotations while $C_{A}^{\mathrm{L}}, C_{A}^{\mathrm{R}}$ must transform as 3 -vectors. The only scalar operators within the $D^{1}$ representation of $\mathcal{U}_{q}\left(\mathrm{su}_{2}\right)$ are multiples of the unit matrix, while every 3 -vector operator is proportional to $\rho^{1}\left(J_{A}\right)$. Hence, up to an overall constant factor our wave equations would be written as

$$
\begin{aligned}
& \left(P^{0}+\alpha_{\mathrm{L}} \rho^{1}\left(J_{A}\right) P^{A}\right) \psi_{\mathrm{L}}=0 \\
& \left(P^{0}+\alpha_{\mathrm{R}} \rho^{1}\left(J_{A}\right) P^{A}\right) \psi_{\mathrm{R}}=0,
\end{aligned}
$$

where $\alpha_{\mathrm{L}}, \alpha_{\mathrm{R}}$ are constants. We determine these constants by considering the wave equations in the momentum eigenspace,

$$
\left(1+\alpha_{\mathrm{L}} \rho^{1}\left(J_{3}\right)\right) \psi_{\mathrm{L}}=0, \quad\left(1+\alpha_{\mathrm{R}} \rho^{1}\left(J_{3}\right)\right) \psi_{\mathrm{R}}=0 .
$$

The space of solutions of each of these equations must equal the image of the projections $\mathbb{P}_{\mathrm{L}}$ and $\mathbb{P}_{\mathrm{R}}$, respectively. This requirement fixes the constants to $\alpha_{\mathrm{L}}=q^{-1}$ and $\alpha_{\mathrm{R}}=-q$.

Although this determines our candidate for the $q$-Maxwell equations, condition (11) has yet to be checked for the boosts. Let $\psi_{0}$ be an element of the momentum eigenspace where $\left(P_{0}, P_{-}, P_{+}, P_{3}\right)=(k, 0,0, k)=:\left(p_{\mu}\right)$. Using the commutation relations between boosts and momentum generators we find [31]

$$
\begin{array}{llrl}
P_{\mu}\left(a \psi_{0}\right) & =q^{-1} p_{\mu}\left(a \psi_{0}\right), & & P_{\mu}\left(b \psi_{0}\right)=q^{-1} p_{\mu}\left(b \psi_{0}\right) \\
P_{\mu}\left(c \psi_{0}\right) & =q p_{\mu}\left(c \psi_{0}\right), & & P_{\mu}\left(d \psi_{0}\right)=q p_{\mu}\left(d \psi_{0}\right) .
\end{array}
$$

By induction it follows, that for any monomial in the boosts, $h=a^{i} b^{j} c^{k} d^{l}$, we have $P_{\mu}\left(h \psi_{0}\right)=q^{k+l-i-j} p_{\mu}\left(h \psi_{0}\right)$. Thus, for $\psi:=h \psi_{0}$, the wave equation (45) takes the form

$$
\left(C_{0}-C_{3}\right) \psi=0 .
$$

Looking separately at the left and right handed part of $\psi=\psi_{\mathrm{L}}+\psi_{\mathrm{R}}$ this equation writes out

$$
\left(\begin{array}{ccc}
0 & 0 & 0 \\
0 & q^{-2} & 0 \\
0 & 0 & q^{-1}[2]
\end{array}\right)\left(\begin{array}{c}
\psi_{\mathrm{L}}^{-} \\
\psi_{\mathrm{L}}^{3} \\
\psi_{\mathrm{L}}^{+}
\end{array}\right)=0,\left(\begin{array}{ccc}
q[2] & 0 & 0 \\
0 & q^{2} & 0 \\
0 & 0 & 0
\end{array}\right)\left(\begin{array}{c}
\psi_{\mathrm{R}}^{-} \\
\psi_{\mathrm{R}}^{3} \\
\psi_{\mathrm{R}}^{+}
\end{array}\right)=0
$$

which is equivalent to $\psi_{\mathrm{L}}^{3}=\psi_{\mathrm{L}}^{+}=0$ and $\psi_{\mathrm{R}}^{-}=\psi_{\mathrm{R}}^{3}=0$. If we now have a solution of Eq. (50), that is, a spinor $\psi$ whose only non-vanishing components are $\psi_{\mathrm{L}}^{-}$and $\psi_{\mathrm{R}}^{+}$, could it happen that by boosting it gets other non-vanishing components, thus turning a solution into a non-solution? The answer to this question is no. 
We exemplify this, applying formula (15) for the action of the boost generator $c$ on a left handed spinor,

$$
\begin{aligned}
c \psi_{\mathrm{L}}^{A}= & \rho^{(1,0)}\left(c_{(1)}\right)_{A^{\prime}}^{A}\left(c_{(2)} \triangleright \psi_{\mathrm{L}}^{A^{\prime}}\right) \\
= & \rho^{(1,0)}(c)^{A}{ }_{A^{\prime}}\left(a \triangleright \psi_{\mathrm{L}}^{A^{\prime}}\right)+\rho^{(1,0)}(d)^{A}{ }_{A^{\prime}}\left(c \triangleright \psi_{\mathrm{L}}^{A^{\prime}}\right) \\
= & -q^{\frac{1}{2}} \lambda \rho^{1}\left(F K^{\frac{1}{2}}\right)^{A}{ }_{A^{\prime}}\left(a \triangleright \psi_{\mathrm{L}}^{A^{\prime}}\right) \\
& \quad+\rho^{1}\left(K^{-\frac{1}{2}}\right)^{A}{ }_{A^{\prime}}\left(c \triangleright \psi_{\mathrm{L}}^{A^{\prime}}\right) \\
= & \left(\begin{array}{c}
-q^{\frac{1}{2}} \lambda[2]^{\frac{1}{2}} a \triangleright \psi_{\mathrm{L}}^{3}+q c \triangleright \psi_{\mathrm{L}}^{-} \\
-q^{\frac{1}{2}} \lambda[2]^{\frac{1}{2}} a \triangleright \psi_{\mathrm{L}}^{+}+c \triangleright \psi_{\mathrm{L}}^{3} \\
q^{-1} c \triangleright \psi_{\mathrm{L}}^{+}
\end{array}\right),
\end{aligned}
$$

which clearly shows that, if $\psi_{\mathrm{L}}^{3}$ and $\psi_{\mathrm{L}}^{+}$vanish, so do $c \psi_{\mathrm{L}}^{3}$ and $c \psi_{\mathrm{L}}^{+}$. Similar calculations can be done for the other boost generators and right handed spinors.

By induction we conclude, that if $\psi_{0}$ is a solution of Eq. (50) and $h=a^{i} b^{j} c^{k} d^{l}$ is a monomial in the boosts, the spinor $\psi=h \psi_{0}$ will be a solution, as well. The algebra of all boosts, $S U_{q}(2)^{\text {op }}$, is generated as linear space by the monomials, thus, $h \psi_{0}$ is a solution for any boost $h \in S U_{q}(2)^{\mathrm{op}}$. Since furthermore every $q$-Lorentz transformation can be written as a sum of products of rotations and boost, $h \psi_{0}$ is a solution for any $q$-Lorentz transformation $h$. We assume that the space of solutions, ker $\mathbb{A}$, is an irreducible representation. This means in particular that the $q$-Lorentz algebra acts transitively on ker $\mathbb{A}$, so any solution can be written as $h \psi_{0}$. Hence, the wave equations

$$
\begin{aligned}
& \rho^{1}\left(J_{A}\right) P^{A} \psi_{\mathrm{L}}=-q P_{0} \psi_{\mathrm{L}} \\
& \rho^{1}\left(J_{A}\right) P^{A} \psi_{\mathrm{R}}=q^{-1} P_{0} \psi_{\mathrm{R}}
\end{aligned}
$$

do indeed satisfy property (11).

Now we want to write these equations as $q$-differential equations $\tilde{C}_{\mu} \partial^{\mu} \psi=0$, where $\tilde{C}_{\mu}$ is defined in Eq. (14). After lengthy calculations we get

$$
\begin{aligned}
& \rho^{1}\left(J_{A^{\prime}}\right)^{B}{ }_{C^{\prime}} \rho^{(1,0)}\left(\left(L_{\mathrm{I}+}^{\Lambda}\right)^{A^{\prime}}{ }_{A}\right)^{C^{\prime}}{ }_{C}=-q^{2} \varepsilon_{C}{ }^{B}{ }_{A} \\
& \rho^{1}\left(J_{A^{\prime}}\right)^{B}{ }_{C^{\prime}} \rho^{(0,1)}\left(\left(L_{\mathrm{I}+}^{\Lambda}\right)^{A^{\prime}}{ }_{A}\right)^{C^{\prime}}{ }_{C}=-q^{-2} \varepsilon_{C}{ }^{B}{ }_{A},
\end{aligned}
$$

so the wave equations (52) can be written as

$$
\vec{\partial} \times \vec{\psi}_{\mathrm{L}}=\mathrm{i} q^{-1} \partial_{0} \vec{\psi}_{\mathrm{L}}, \quad \vec{\partial} \times \vec{\psi}_{\mathrm{R}}=-\mathrm{i} q \partial_{0} \vec{\psi}_{\mathrm{R}}
$$

where $\vec{\psi}_{\mathrm{R}}=\left(\psi_{\mathrm{R}}^{A}\right), \vec{\psi}_{\mathrm{L}}=\left(\psi_{\mathrm{L}}^{A}\right)$ and where the cross product is defined in Eq. (73). A spinor $\vec{\psi}_{\mathrm{L}}$ which is a solution to this equation must also satisfy the mass zero condition. Using identities (174) for the cross product, commutation relations (77) of the derivations, $\vec{\partial} \times \vec{\partial}=-i \lambda \partial_{0} \vec{\partial}$, and the wave equation (54), we rewrite the 
mass zero condition as

$$
\begin{aligned}
0 & =\partial_{\mu} \partial^{\mu} \vec{\psi}_{\mathrm{L}}=\left(\partial_{0}^{2}-\vec{\partial} \cdot \vec{\partial}\right) \vec{\psi}_{\mathrm{L}} \\
& =\partial_{0}^{2} \vec{\psi}_{\mathrm{L}}-(\vec{\partial} \times \vec{\partial}) \times \vec{\psi}_{\mathrm{L}}+\vec{\partial} \times\left(\vec{\partial} \times \vec{\psi}_{\mathrm{L}}\right)-\vec{\partial}\left(\vec{\partial} \cdot \vec{\psi}_{\mathrm{L}}\right) \\
& =\partial_{0}^{2} \vec{\psi}_{\mathrm{L}}+\mathrm{i} \lambda \partial_{0}\left(\vec{\partial} \times \vec{\psi}_{\mathrm{L}}\right)+\vec{\partial} \times\left(\mathrm{i} q^{-1} \partial_{0} \vec{\psi}_{\mathrm{L}}\right)-\vec{\partial}\left(\vec{\partial} \cdot \vec{\psi}_{\mathrm{L}}\right) \\
& =\partial_{0}^{2} \vec{\psi}_{\mathrm{L}}-q^{-1} \lambda \partial_{0}^{2} \vec{\psi}_{\mathrm{L}}-q^{-2} \partial_{0}^{2} \vec{\psi}_{\mathrm{L}}-\vec{\partial}\left(\vec{\partial} \cdot \vec{\psi}_{\mathrm{L}}\right) \\
& =-\vec{\partial}\left(\vec{\partial} \cdot \vec{\psi}_{\mathrm{L}}\right) .
\end{aligned}
$$

Contracting the wave equation with $\vec{\partial}$

$$
\begin{aligned}
\vec{\partial} \cdot\left(\vec{\partial} \times \vec{\psi}_{\mathrm{L}}\right) & =(\vec{\partial} \times \vec{\partial}) \cdot \vec{\psi}_{\mathrm{L}}=-\mathrm{i} \lambda \partial_{0}\left(\vec{\partial} \cdot \vec{\psi}_{\mathrm{L}}\right) \\
& =\mathrm{i} q^{-1} \partial_{0}\left(\vec{\partial} \cdot \vec{\psi}_{\mathrm{L}}\right),
\end{aligned}
$$

we see that $\partial_{0}\left(\vec{\partial} \cdot \vec{\psi}_{\mathrm{L}}\right)=0$ if $\vec{\psi}_{\mathrm{L}}$ is to satisfy the wave equation. Together with Eq. (55) this means that the mass zero condition is equivalent to $\partial_{\mu}\left(\vec{\partial} \cdot \vec{\psi}_{\mathrm{L}}\right)=0$, that is, $\vec{\partial} \cdot \vec{\psi}_{\mathrm{L}}$ must be a constant number. In a momentum eigenspace we have $\partial_{0}\left(\vec{\partial} \cdot \vec{\psi}_{\mathrm{L}}\right)=k\left(\vec{\partial} \cdot \vec{\psi}_{\mathrm{L}}\right)$, so this constant number must be zero. The same reasoning applies to the right handed spinor $\vec{\psi}_{\mathrm{R}}$.

We conclude that the wave equations (54) together with the mass zero condition $\partial_{\mu} \partial^{\mu} \psi=0$ are equivalent to

$$
\begin{array}{ll}
\vec{\partial} \times \vec{\psi}_{\mathrm{L}}=\mathrm{i} q^{-1} \partial_{0} \vec{\psi}_{\mathrm{L}}, & \vec{\partial} \cdot \vec{\psi}_{\mathrm{L}}=0 \\
\vec{\partial} \times \vec{\psi}_{\mathrm{R}}=-\mathrm{i} q \partial_{0} \vec{\psi}_{\mathrm{R}}, & \vec{\partial} \cdot \vec{\psi}_{\mathrm{R}}=0,
\end{array}
$$

which we will call the $q$-Maxwell equations.

\subsection{The $q$-Electromagnetic Field}

Finally, we write the $q$-Maxwell equations in a more familiar form, that is, in terms of the $q$-deformed electric and magnetic fields. In the undeformed case the electric and magnetic fields can - up to constant factors - be characterized within the $D^{(1,0)} \oplus D^{(0,1)}$ representation as eigenstates of the parity operator (19). The electric field should transform like a polar vector $\mathcal{P} \vec{E}=-\vec{E}$, while the magnetic field must be an axial vector $\mathcal{P} \vec{B}=\vec{B}$. Recall, that the parity operator $\mathcal{P}$ acts on $q$-spinors by exchanging the left and the right handed parts $\mathcal{P} \psi_{\mathrm{L}}=\psi_{\mathrm{R}}$, $\mathcal{P} \psi_{\mathrm{R}}=\psi_{\mathrm{L}}$. This fixes the fields

$$
\vec{E}=\mathrm{i}\left(\vec{\psi}_{\mathrm{R}}-\vec{\psi}_{\mathrm{L}}\right), \quad \vec{B}=\vec{\psi}_{\mathrm{R}}+\vec{\psi}_{\mathrm{L}}
$$

up to constant factors which have been chosen to give the right undeformed limit. Spinor conjugation of the fields is now the same as ordinary conjugation 
$\bar{E}^{A}=\left(E^{A}\right)^{*}, \bar{B}^{A}=\left(B^{A}\right)^{*}$. In terms of these fields, the $q$-Maxwell equations (57) take the form

$$
\begin{array}{ll}
\vec{\partial} \times \vec{E}=\frac{1}{2}[2] \partial_{0} \vec{B}-\frac{1}{2} \mathrm{i} \lambda \partial_{0} \vec{E}, & \vec{\partial} \cdot \vec{E}=0 \\
\vec{\partial} \times \vec{B}=-\frac{1}{2}[2] \partial_{0} \vec{E}-\frac{1}{2} \mathrm{i} \lambda \partial_{0} \vec{B}, & \vec{\partial} \cdot \vec{B}=0 .
\end{array}
$$

We can also express the $q$-Maxwell equations in terms of a field strength tensor $F^{\mu \nu}$. According to the Clebsch-Gordan series (76) we can embed, say, a $D^{(1,0)}$ representation in to a $D^{\left(\frac{1}{2}, \frac{1}{2}\right)} \otimes D^{\left(\frac{1}{2}, \frac{1}{2}\right)}$ representation. Explicitly, a basis $\left\{e_{C}\right\}$ of the former is mapped to a basis $\left\{e_{\mu}^{\prime} \otimes e_{\nu}^{\prime}\right\}$ of the latter by 31]

$$
e_{C} \mapsto e_{A}^{\prime} \otimes e_{B}^{\prime} \varepsilon_{C}^{A B}+q e_{0}^{\prime} \otimes e_{C}^{\prime}-q^{-1} e_{C}^{\prime} \otimes e_{0}^{\prime} .
$$

Accordingly, we map a left 3-vector

$$
\psi_{\mathrm{L}}=e_{C} \otimes \psi_{\mathrm{L}}^{C} \mapsto\left(e_{\mu}^{\prime} \otimes e_{\nu}^{\prime}\right) \otimes F_{\mathrm{L}}^{\mu \nu},
$$

where

$$
F_{\mathrm{L}}^{\mu \nu}:=\left(\begin{array}{cc}
F_{\mathrm{L}}^{00} & F_{\mathrm{L}}^{0 N} \\
F_{\mathrm{L}}^{M 0} & F_{\mathrm{L}}^{M N}
\end{array}\right)=\left(\begin{array}{cc}
0 & q \psi_{\mathrm{L}}^{N} \\
-q^{-1} \psi_{\mathrm{L}}^{M} & \varepsilon^{M N}{ }_{C} \psi_{\mathrm{L}}^{C}
\end{array}\right),
$$

and where $M, N$ run through $\{-,+, 3\}$. In the same manner we obtain for the right handed part

$$
F_{\mathrm{R}}^{\mu \nu}:=\left(\begin{array}{cc}
0 & -q^{-1} \psi_{\mathrm{R}}^{N} \\
q \psi_{\mathrm{R}}^{M} & \varepsilon^{M N}{ }_{C} \psi_{\mathrm{R}}^{C}
\end{array}\right) .
$$

In terms of these matrices the $q$-Maxwell equations (157) take the form $\partial_{\nu} F_{\mathrm{L}}^{\mu \nu}=0$ and $\partial_{\nu} F_{\mathrm{R}}^{\mu \nu}=0$. By construction, we have $\mathbb{P}_{(1,0) \sigma \tau}^{\mu \nu} F_{\mathrm{L}}^{\sigma \tau}=F_{\mathrm{L}}^{\mu \nu}$ and $\mathbb{P}_{(0,1) \sigma \tau}^{\mu \nu} F_{\mathrm{R}}^{\sigma \tau}=$ $F_{\mathrm{R}}^{\mu \nu}$. This suggests to introduce the field strength tensor and its dual

$$
F^{\mu \nu}:=\mathrm{i}\left(F_{\mathrm{L}}^{\mu \nu}+F_{\mathrm{R}}^{\mu \nu}\right), \quad \tilde{F}^{\mu \nu}:=\mathrm{i}\left(F_{\mathrm{L}}^{\mu \nu}-F_{\mathrm{R}}^{\mu \nu}\right),
$$

for which we have

$$
F^{\mu \nu}=\mathbb{P}_{\mathrm{A} \sigma \tau}^{\mu \nu} F^{\sigma \tau}, \quad \tilde{F}^{\mu \nu}=\varepsilon_{\sigma \tau}^{\mu \nu} F^{\sigma \tau},
$$

where the $q$-epsilon tensor is commonly defined as $\varepsilon^{\mu \nu}{ }_{\sigma \tau}=\mathbb{P}_{(1,0) \sigma \tau}^{\mu \nu}-\mathbb{P}_{(0,1) \sigma \tau}^{\mu \nu}$. In terms of the electric and the magnetic field this is

$$
\begin{aligned}
F^{\mu \nu} & :=\left(\begin{array}{cc}
0 & -\frac{1}{2}[2] E^{N}+\frac{1}{2} \mathrm{i} \lambda B^{N} \\
\frac{1}{2}[2] E^{M}+\frac{1}{2} \mathrm{i} \lambda B^{M} & \mathrm{i} \varepsilon^{M N}{ }_{C} B^{C}
\end{array}\right) \\
\tilde{F}^{\mu \nu} & :=\left(\begin{array}{cc}
0 & \frac{1}{2}[2] \mathrm{i} B^{N}-\frac{1}{2} \lambda E^{N} \\
-\frac{1}{2}[2] \mathrm{i} B^{M}-\frac{1}{2} \lambda E^{M} & -\varepsilon^{M N}{ }_{C} E^{C}
\end{array}\right) .
\end{aligned}
$$

Finally, the $q$-Maxwell equations take the form

$$
\partial_{\nu} F^{\mu \nu}=0, \quad \partial_{\nu} \tilde{F}^{\mu \nu}=0,
$$

in complete analogy to the undeformed case. 


\section{Conclusion}

In this paper the free Dirac equation and the free Maxwell equations on quantum Minkowski space have been determined. We started from the restriction on rest states (or certain light cone states for the massless case), requiring $q$-Lorentz covariance, effectively "boosting" the wave equations. For his method the explicit action of the momenta on $q$-spinors was not needed and, consequently, was not constructed. This approach makes the calculations to a large extent independent of the explicit realization of the momenta. It is in complete analogy to the classic methods for the undeformed case [30] and parallels the construction of induced representations of the Poincaré algebra.

The natural next step is to construct the action of the $q$-Poincaré algebra on $q$-spinors, explicitly, as $q$-differential operators on the $q$-Minkowski algebra of noncommutative wave functions. Then, the wave equations obtained here can be interpreted as q-differential equations, their solutions can be calculated, and the generalized Cauchy problem can be studied. This has already been done and will be published elsewhere.

The free $q$-Dirac equation obtained here is a good starting point for the construction of the $q$-Dirac equation with electromagnetic interaction. In fact, the methods to introduce gauge interactions developed in [4,5] are quite general and do apply here, although it might be necessary to add a vielbein formalism [35]. Judging from what is known about models on spaces of constant noncommutativity (as mentioned in the introduction) it is not unreasonable to expect the ensuing model on quantum Minkowski space to possess a rich phenomenology. The investigation of this phenomenology is expected to be a computational challenge, though.

On of the main hopes and, at the same time, open questions in noncommutative geometry is, whether noncommutativity can smoothen the divergences of quantum field theory. While it would seem bold to claim that noncommutativity will regularize quantum field theory, there are some indirect indications that it might weaken the divergences and improve renormalizability. For example, the spectra of space-time observables on quantum spaces exhibit a natural lattice structure [36]. We plan to approach this question by calculating the Green's functions of the free $q$-wave equations, since those would appear as bare propagators in loop calculations of QED on quantum Minkowski space. Studying the divergences of free propagators on quantum Minkowski space would be a well defined

and rigorous test of the conjectured regularizing properties of noncommutative geometry.

\section{Acknowledgements}

I would like to thank Julius Wess, Peter Schupp, and Fabian Bachmaier for helpful comments. This work was supported by the Studienstiftung des deutschen Volkes. 


\section{A Useful Formulas}

Let $E, F, K$, and $K^{-1}$ be the generators of $\mathcal{U}_{q}\left(\mathrm{su}_{2}\right)$. The set of generators $\left\{J_{A}\right\}=\left\{J_{-}, J_{3}, J_{+}\right\}$of $\mathcal{U}_{q}\left(\mathrm{su}_{2}\right)$ defined as

$$
\begin{aligned}
J_{-} & :=q[2]^{-\frac{1}{2}} K F \\
J_{3} & :=[2]^{-1}\left(q^{-1} E F-q F E\right) \\
J_{+} & :=-[2]^{-\frac{1}{2}} E
\end{aligned}
$$

is the left 3 -vector operator of angular momentum. The center of $\mathcal{U}_{q}\left(\mathrm{su}_{2}\right)$ is generated by

$$
W:=K-\lambda J_{3}=K-\lambda[2]^{-1}\left(q^{-1} E F-q F E\right),
$$

the Casimir operator of angular momentum. $W$ is related to $J_{A}$ by

$$
W^{2}-1=\lambda^{2}\left(J_{3}^{2}-q^{-1} J_{-} J_{+}-q J_{+} J_{-}\right)=\lambda^{2} J_{A} J_{B} g^{A B},
$$

thus defining the 3-metric $g^{A B}$, by which we raise 3 -vector indices $X^{A}=g^{A B} X_{B}$. There is also an $\varepsilon$-tensor

$$
\begin{array}{lll}
\varepsilon^{-3}{ }_{-}=q^{-1} & \varepsilon^{3-}=-q & \\
\varepsilon^{-+}{ }_{3}=1 & \varepsilon^{+-}{ }_{3}=-1 & \varepsilon^{33}{ }_{3}=-\lambda \\
\varepsilon^{3+}{ }_{+}=q^{-1} & \varepsilon^{+3}{ }_{+}=-q, &
\end{array}
$$

so we can define a scalar and a vector product by

$$
\vec{X} \cdot \vec{Y}:=g^{A B} X_{A} Y_{B}, \quad(\vec{X} \times \vec{Y})_{C}:=\mathrm{i} X_{A} Y_{B} \varepsilon_{C}^{A B},
$$

for which we have the useful identities

$$
\begin{gathered}
\vec{X} \cdot(\vec{Y} \times \vec{Z})=(\vec{X} \times \vec{Y}) \cdot \vec{Z} \\
(\vec{X} \times \vec{Y}) \times \vec{Z}-(\vec{X} \cdot \vec{Y}) \vec{Z}=\vec{X} \times(\vec{Y} \times \vec{Z})-\vec{X}(\vec{Y} \cdot \vec{Z}) .
\end{gathered}
$$

Let $\left(\begin{array}{ll}a & b \\ c & d\end{array}\right)$ be the matrix of generators of $S U_{q}(2)^{\text {op }}$, the opposite algebra of the quantum group $S U_{q}(2)$. The Hopf-* algebra generated by the Hopf-* subalgebras $\mathcal{U}_{q}\left(\mathrm{su}_{2}\right)$ and $S U_{q}(2)^{\text {op }}$ with cross commutation relations

$$
\begin{aligned}
& \left(\begin{array}{ll}
a & b \\
c & d
\end{array}\right) E=\left(\begin{array}{cc}
q E a-q^{\frac{3}{2}} b & q^{-1} E b \\
q E c+q^{\frac{3}{2}} K a-q^{\frac{3}{2}} d & q^{-1} E d+q^{-\frac{1}{2}} K b
\end{array}\right) \\
& \left(\begin{array}{ll}
a & b \\
c & d
\end{array}\right) F=\left(\begin{array}{cc}
q F a+q^{-\frac{1}{2}} c & q F b-q^{-\frac{1}{2}} K^{-1} a+q^{-\frac{1}{2}} d \\
q^{-1} F c & q^{-1} F d-q^{-\frac{5}{2}} K^{-1} c
\end{array}\right) \\
& \left(\begin{array}{ll}
a & b \\
c & d
\end{array}\right) K=K\left(\begin{array}{cc}
a & q^{-2} b \\
q^{2} c & d
\end{array}\right),
\end{aligned}
$$


which is the Drinfeld double of $\mathcal{U}_{q}\left(\mathrm{su}_{2}\right)$ and $S U_{q}(2)^{\text {op }}$, is the $\boldsymbol{q}$-Lorentz algebra $\mathcal{H}=\mathcal{U}_{q}\left(\mathrm{sl}_{2}(\mathbb{C})\right)$ [37].

Other forms of the $q$-Lorentz algebra can be found in the literature [38, 32,39], which are essentially equivalent [40,31. Very useful for the representation theory is the form where $\mathcal{U}_{q}\left(\mathrm{sl}_{2}(\mathbb{C})\right) \cong \mathcal{U}_{q}\left(\mathrm{sl}_{2}\right) \otimes \mathcal{U}_{q}\left(\mathrm{sl}_{2}\right)$ as algebra. This isomorphism is defined on rotations $l \in \mathcal{U}_{q}\left(\mathrm{su}_{2}\right)$ by the coproduct $l \mapsto \Delta(l) \in \mathcal{U}_{q}\left(\mathrm{sl}_{2}\right) \otimes \mathcal{U}_{q}\left(\mathrm{sl}_{2}\right)$ and for the generators of boosts as

$$
\begin{aligned}
a & \mapsto K^{\frac{1}{2}} \otimes K^{-\frac{1}{2}} \\
b & \mapsto q^{-\frac{1}{2}} \lambda K^{\frac{1}{2}} \otimes K^{-\frac{1}{2}} E \\
c & \mapsto-q^{\frac{1}{2}} \lambda F K^{\frac{1}{2}} \otimes K^{-\frac{1}{2}} \\
d & \mapsto K^{-\frac{1}{2}} \otimes K^{\frac{1}{2}}-\lambda^{2} F K^{\frac{1}{2}} \otimes K^{-\frac{1}{2}} E .
\end{aligned}
$$

On this form of the $q$-Lorentz algebra the representation maps of the irreducible representations are $\rho^{j_{1}} \otimes \rho^{j_{2}}$, where $\rho^{j}$ is the spin- $j$ representation of $\mathcal{U}_{q}\left(\mathrm{sl}_{2}\right)$. For the 4 -vector representation we have the usual Clebsch-Gordan series

$$
D^{\left(\frac{1}{2}, \frac{1}{2}\right)} \otimes D^{\left(\frac{1}{2}, \frac{1}{2}\right)} \cong D^{(0,0)} \oplus D^{(1,0)} \oplus D^{(0,1)} \oplus D^{(1,1)} .
$$

The projection matrices on the according subspaces are denoted by $\mathbb{P}_{(0,0)}, \mathbb{P}_{(1,0)}$, $\mathbb{P}_{(0,1)}, \mathbb{P}_{(1,1)}$, the $q$-antisymmetrizer by $\mathbb{P}_{\mathrm{A}}:=\mathbb{P}_{(1,0)}+\mathbb{P}_{(0,1)}$, and the $q$-symmetrizer by $\mathbb{P}_{\mathrm{S}}:=\mathbb{P}_{(0,0)}+\mathbb{P}_{(1,1)}=1-\mathbb{P}_{\mathrm{A}}$. With respect to the 4 -vector basis

\begin{tabular}{c|ccc} 
& \multicolumn{3}{|c}{$[2]^{2}\left(\mathbb{P}_{\mathrm{A}}\right)^{a b}{ }_{c d}=$} \\
& $C 0$ & $0 D$ & $C D$ \\
\hline$A 0$ & $2 \delta_{C}^{A}$ & $-[4][2]^{-1} \delta_{D}^{A}$ & $\lambda \varepsilon_{C}{ }^{A}{ }_{D}$ \\
$0 B$ & $-[4][2]^{-1} \delta_{C}^{B}$ & $2 \delta_{D}^{B}$ & $\lambda \varepsilon_{C}{ }^{B}{ }_{D}$ \\
$A B$ & $-\lambda \varepsilon^{A B}{ }_{C}$ & $-\lambda \varepsilon^{A B}{ }_{D}$ & $2 \varepsilon^{A B}{ }_{X} \varepsilon_{C}{ }^{X}{ }_{D}$
\end{tabular}

where $A, B, C, D$ run through $\{-,+, 3\} . \mathcal{U}_{q}\left(\operatorname{sl}_{2}(\mathbb{C})\right)$ possesses two universal $\mathcal{R}$ matrices, one of which is antireal $\mathcal{R}^{* \otimes *}=\mathcal{R}^{-1}$, the other one is real $\mathcal{R}^{* \otimes *}=\mathcal{R}_{21}$.

The $*$-algebra generated by $P_{0}, P_{-}, P_{+}, P_{3}$ with commutation relations

$$
P_{0} P_{A}=P_{A} P_{0}, \quad P_{A} P_{B} \varepsilon^{A B}{ }_{C}=-\lambda P_{0} P_{C},
$$

and *-structure $P_{0}^{*}=P_{0}, P_{-}^{*}=-q^{-1} P_{+}, P_{+}^{*}=-q P_{-}, P_{3}^{*}=P_{3}$ is the $\boldsymbol{q}$ Minkowski space algebra $\mathcal{X}=\mathbb{R}_{q}^{1,3}$. The center of $\mathbb{R}_{q}^{1,3}$ is generated by

$$
m^{2}:=P_{\mu} P_{\nu} \eta^{\mu \nu}=P_{0}^{2}+q^{-1} P_{-} P_{+}+q P_{+} P_{-}-P_{3}^{2},
$$

the mass Casimir, thus defining the 4-metric $\eta^{\mu \nu}$. It is related to the 3-metric by $\eta^{A B}=-g^{A B}$ for $A, B \in\{-,+, 3\}$. The generators $P_{\mu}$ carry a 4 -vector representation of $\mathcal{H}$ which in this particular basis is denoted by $h \triangleright P_{\nu}=P_{\mu} \Lambda(h)^{\nu}{ }_{\mu}$. It turns $\mathcal{X}$ into a $\mathcal{H}$-module $*$-algebra. 
The $\boldsymbol{q}$-Poincaré algebra $\mathcal{A}$ is the $*$-algebra generated by the $q$-Lorentz algebra $\mathcal{H}=\mathcal{U}_{q}\left(\mathrm{sl}_{2}(\mathbb{C})\right)$ and the $q$-Minkowski algebra $\mathcal{X}=\mathbb{R}_{q}^{1,3}$ with cross commutation relations

$$
h P_{\nu}=P_{\mu} \Lambda\left(h_{(1)}\right)_{\nu}^{\mu} h_{(2)},
$$

for all $h \in \mathcal{H}$. In other words, $\mathcal{A}$ is the Hopf semidirect product $\mathcal{A}=\mathcal{X} \rtimes \mathcal{H}$.

The left Hopf adjoint action of $\mathcal{H}$ on $\mathcal{A}$ is defined as

$$
\operatorname{ad}_{\mathrm{L}} h \triangleright a:=h_{(1)} a S\left(h_{(2)}\right) .
$$

The commutation relations (179) are precisely such that the left Hopf adjoint action equals the 4 -vector action $\operatorname{ad}_{\mathrm{L}} h \triangleright P_{\nu}=P_{\mu} \Lambda(h)_{\nu}^{\mu}$. Any set of operators with this property will be called a 4 -vector operator. In particular we have

$$
\begin{aligned}
P_{-} & =\operatorname{ad}_{\mathrm{L}}\left(-q^{-\frac{1}{2}} \lambda^{-1}[2]^{\frac{1}{2}} c\right) \triangleright P_{0} \\
P_{+} & =\operatorname{ad}_{\mathrm{L}}\left(q^{\frac{1}{2}} \lambda^{-1}[2]^{\frac{1}{2}} b\right) \triangleright P_{0} \\
P_{3} & =\operatorname{ad}_{\mathrm{L}}\left(\lambda^{-1}(d-a)\right) \triangleright P_{0},
\end{aligned}
$$

so, if we know the zero component of a 4 -vector operator, we can easily compute the other components.

\section{B Gamma matrices for the real R-matrix}

If the real $\mathcal{R}$-matrix instead of the antireal one only the expressions for the $\gamma$ matrices change slightly. We now have to observe that the different projector decomposition of the $R$-matrix [39, 31] leads to $\gamma_{\mu} \gamma_{\nu} P^{\mu} P^{\nu}=q^{3} P_{\mu} P^{\mu}$, which differs from the antireal case by the factor $q^{3}$. This implies $\left(\gamma_{0}\right)^{2}=q^{3}$. Eq. (30) now becomes

$$
\gamma_{0}=q^{\frac{3}{2}}\left(\begin{array}{ll}
0 & 1 \\
1 & 0
\end{array}\right), \quad \gamma_{A}=q^{\frac{3}{2}}\left(\begin{array}{cc}
0 & q \sigma_{A} \\
-q^{-1} \sigma_{A} & 0
\end{array}\right)
$$

Eq. (31) reads

$$
\gamma_{0}^{(j)}=q^{\frac{3}{2}}\left(\begin{array}{ll}
0 & 1 \\
1 & 0
\end{array}\right), \gamma_{A}^{(j)}=q^{\frac{3}{2}}[2]\left(\begin{array}{cc}
0 & q \rho^{j}\left(J_{A}\right) \\
-q^{-1} \rho^{j}\left(J_{A}\right) & 0
\end{array}\right),
$$

and, finally, Eq. (33) has to be replaced by

$$
\tilde{\gamma}_{0}=\left(\begin{array}{cc}
0 & q^{\frac{3}{2}} \\
q^{-\frac{3}{2}} & 0
\end{array}\right), \quad \tilde{\gamma}_{A}=\left(\begin{array}{cc}
0 & q^{\frac{1}{2}} \tilde{\sigma}_{A} \\
-q^{-\frac{1}{2}} \tilde{\sigma}_{A} & 0
\end{array}\right) .
$$

All other results remain unchanged. 


\section{References}

[1] M. R. Douglas and N. A. Nekrasov, "Noncommutative field theory," Rev. Mod. Phys. 73 (2001) 977-1029, hep-th/0106048.

[2] R. J. Szabo, "Quantum field theory on noncommutative spaces," Phys. Rept. 378 (2003) 207-299, hep-th/0109162.

[3] N. Seiberg and E. Witten, "String theory and noncommutative geometry," JHEP 09 (1999) 032, hep-th/9908142.

[4] J. Madore, S. Schraml, P. Schupp, and J. Wess, "Gauge theory on noncommutative spaces," Eur. Phys. J. C16 (2000) 161-167, hep-th/0001203.

[5] B. Jurco, L. Moller, S. Schraml, P. Schupp, and J. Wess, "Construction of non-Abelian gauge theories on noncommutative spaces," Eur. Phys. J. C21 (2001) 383-388, hep-th/0104153.

[6] X. Calmet, B. Jurco, P. Schupp, J. Wess, and M. Wohlgenannt, "The standard model on non-commutative space-time," Eur. Phys. J. C23 (2002) 363-376, hep-ph/0111115.

[7] E. O. Iltan, "The noncommutative effects on the dipole moments of fermions in the standard model," JHEP 05 (2003) 065, hep-ph/0304097.

[8] P. Minkowski, P. Schupp, and J. Trampetic, "Non-commutative '*-charge radius' and '*-dipole moment' of the neutrino," hep-th/0302175

[9] P. Schupp, J. Trampetic, J. Wess, and G. Raffelt, "The photon neutrino interaction in non-commutative gauge field theory and astrophysical bounds," hep-ph/0212292.

[10] OPAL Collaboration, G. Abbiendi et al., "Test of non-commutative QED in the process $\mathrm{e}^{+} \mathrm{e}^{-} \rightarrow \gamma \gamma$ at LEP," hep-ex/0303035.

[11] I. Hinchliffe and N. Kersting, "Review of the phenomenology of noncommutative geometry," hep-ph/0205040.

[12] S. Minwalla, M. Van Raamsdonk, and N. Seiberg, "Noncommutative perturbative dynamics," JHEP 02 (2000) 020, hep-th/9912072.

[13] A. Matusis, L. Susskind, and N. Toumbas, "The IR/UV connection in the non-commutative gauge theories," JHEP 12 (2000) 002, hep-th/0002075.

[14] L. Alvarez-Gaume and M. A. Vazquez-Mozo, "General properties of noncommutative field theories," hep-th/0305093. 
[15] D. J. Bird et al., "Detection of a cosmic ray with measured energy well beyond the expected spectral cutoff due to comic microwave radiation," Astrophys. J. 441 (1995) 144-150.

[16] G. Amelino-Camelia, "Relativity in space-times with short-distance structure governed by an observer-independent (Planckian) length scale," Int. J. Mod. Phys. D11 (2002) 35-60, gr-qc/0012051.

[17] G. Amelino-Camelia, "Doubly-Special Relativity: First Results and Key Open Problems," Int. J. Mod. Phys. D11 (2002) 1643, gr-qc/0210063.

[18] A. Agostini, G. Amelino-Camelia, and F. D'Andrea, "Hopf-algebra description of noncommutative-spacetime symmetries," hep-th/0306013.

[19] H. Wachter and M. Wohlgenannt, "*-Products on Quantum Spaces," Eur. Phys. J. C23 (2002) 761-767, hep-th/0103120.

[20] C. Blohmann, "Covariant realization of quantum spaces as star products by Drinfeld twists," math.qa/0209180, to appear in J. Math. Phys. 10 (2003).

[21] L. Mesref, "A map between q-deformed noncommutative and ordinary gauge theories," hep-th/0209005.

[22] E. P. Wigner, "On Unitary Representations of the Inhomogeneous Lorentz Group," Annals Math. 40 (1939) 149-204.

[23] O. Ogievetskii, W. B. Schmidke, J. Wess, and B. Zumino, " $q$-Deformed Poincaré algebra," Commun. Math. Phys. 150 (1992) 495.

[24] V. K. Dobrev, "New q-Minkowski space-time and q-Maxwell equations hierarchy from q-conformal invariance," Phys. Lett. B341 (1994) 133-138.

[25] A. Schirrmacher, "Quantum groups, quantum space-time, and Dirac equation,". Talk given at NATO Advanced Research Workshop on Low Dimensional Topology and Quantum Field Theory, Cambridge, England, 6-13 Sep 1992.

[26] M. Pillin, "q-deformed relativistic wave equations," J. Math. Phys. 35 (1994) 2804-2817, hep-th/9310097.

[27] X.-C. Song, "Covariant differential calculus on quantum Minkowski space and the q-analog of Dirac equation," Z. Phys. C55 (1992) 417-422.

[28] U. Meyer, "Wave equations on q-Minkowski space," Commun. Math. Phys. 174 (1995) 457-476, hep-th/9404054. 
[29] P. Podles, "Solutions of Klein-Gordon and Dirac equations on quantum Minkowski spaces," Commun. Math. Phys. 181 (1996) 569-586, q-alg/9510019.

[30] A. O. Barut and R. Raczka, Theory of Group Representations and Applications. PWN-Polish Scientific Publishers, 1977.

[31] C. Blohmann, Spin Representations of the q-Poincaré Algebra. PhD thesis, Ludwig-Maximilians-Universität München, 2001. math.qa/0110219.

[32] S. Majid, "Braided momentum in the q-Poincare group," J. Math. Phys. 34 (1993) 2045-2058, hep-th/9210141.

[33] S. Majid, Foundations of Quantum Group Theory. Cambridge Univ. Press, 1995.

[34] C. Blohmann, "Spin in the q-Deformed Poincaré Algebra," math.qa/0111008, to be published in Commun. Math. Phys.

[35] S. Schraml, "Non-Abelian gauge theory on q-quantum spaces," hep-th/0208173.

[36] M. Fichtmüller, A. Lorek, and J. Wess, "q-deformed Phase Space and its Lattice Structure," Z. Phys. C71 (1996) 533-538, hep-th/9511106.

[37] P. Podles and S. L. Woronowicz, "Quantum deformation of Lorentz group," Commun. Math. Phys. 130 (1990) 381.

[38] O. Ogievetskii, W. B. Schmidke, J. Wess, and B. Zumino, "Six generator q-deformed Lorentz algebra," Lett. Math. Phys. 23 (1991) 233-240.

[39] A. Lorek, W. Weich, and J. Wess, "Non-commutative Euclidean and Minkowski structures," Z. Phys. C76 (1997) 375.

[40] M. Rohregger and J. Wess, " $q$-deformed Lorentz-algebra in Minkowski phase space," Eur. Phys. J. C7 (1999), no. 1, 177-183. 